\title{
Carotid Artery Diameters, Carotid Endarterectomy Techniques and Restenosis
}

\author{
Giovanni Bertoletti ${ }^{1}$, Alessandro Varroni ${ }^{1}$, Maria Misuraca ${ }^{1}$, Marco Massucci ${ }^{1}$, Antonio Pacelli ${ }^{2}$, Marco Ciacciarelli ${ }^{2}$ and Luigi luliano ${ }^{2 *}$ \\ ${ }^{1}$ Division of Vascular Surgery, Goretti Hospital, Latina, Italy \\ ${ }^{2}$ Department of Medico-Surgical Sciences and Biotechnology, Vascular Biology, Atherothrombosis \& Mass Spectrometry Lab, Sapienza University of Rome at Latina, Italy
}

\begin{abstract}
Background: Restenosis of the carotid artery is a major complication of carotid endarterectomy (CEA). The purpose of this study was to examine the role of CEA techniques on carotid dimensions variation, postoperative versus preoperative multi-segmental diameters and its impact on the development of restenosis at 12 months follow up.

Methods: 175 consecutive patients eligible for carotid surgery were included in the study. 75 underwent CEA by patch reconstruction (PR), 53 by eversion (EV) and 47 by primary closure (PC). Before the procedures and at discharge, carotid diameters were measured at four reference points (common carotid, CC; carotid bulb, CB; proximal internal carotid artery, PICA; distal internal carotid artery, DICA) by ultrasonography. The rate of minor (< $50 \%$ ) and major ( $\geq 50 \%$ ) restenosis was evaluated at 12 months follow up.

Results: PR produced an increase in all carotid diameters while PC and EV produced a decrease in carotid diameters, having PC affected all diameters while EV affected CB and PICA diameter. However, postoperative diameters had comparable dimension independently of the surgical technique used. The rate of overall and major restenosis did not differ significantly between the three types of surgery. Logistic regression analysis showed

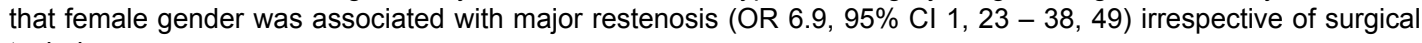
technique.
\end{abstract}

Conclusion: This study shows that carotid diameters and restenosis rate after CEA are comparable whatever is the surgical technique adopted, and that women are at high risk of major restenosis.

\section{Keywords: CEA; ICA; PICA diameter}

\section{Introduction}

Carotid atherosclerosis plays a striking role in the development of ischemic stroke that is the third leading cause of death, and one of the main causes of disability, in western countries. Atherosclerosis, a chronic and progressive disease of the arterial wall which is preferentially located at the carotid bifurcation and extending into the Internal Carotid Artery (ICA), is complicated by in situ plaque thrombosis and downstream embolism-dependent cerebral ischemia. Treatment of carotid atherosclerosis is based on surgical endarterectomy and endovascular procedures, including transluminal balloon angioplasty or stenting. Carotid endarterectomy is one of the most common non-cardiac vascular operations performed in western countries and is an effective and relatively safe procedure for the treatment of atherosclerotic disease involving carotid bifurcation. The goal of endarterectomy is to remove an obstructing or embolic lesion and reconstruct a durable arterial segment free of flow abnormality. Techniques used for carotid endarterectomy (CEA) include primary closure (PC), patch reconstruction (PR), and eversion (EV). PC and $\mathrm{PR}$ employ a longitudinal arteriotomy up the internal carotid artery, EV technique involves the oblique transection of the internal carotid artery at its origin in the carotid bulb, followed by removal of the plaque using the eversion maneuver [1]. Several clinical trials have demonstrated the efficacy of CEA in preventing stroke and transient ischemic attacks (TIA) in both symptomatic and asymptomatic patients with flow-limiting lesions [2-5]. The dramatic increase in the number of carotid endarterectomies performed in both symptomatic and asymptomatic patients has appropriately provoked an increased attention in documenting the efficacy and durability of this procedure. Compared to endarterectomy, restenosis is more common in patients assigned to endovascular treatment of carotid stenosis by percutaneous transluminal balloon angioplasty or insertion of a stent. The Carotid and Vertebral Artery Transluminal Angioplasty Study (CAVATAS) [6] showed that carotid restenosis or occlusion was about three times higher after endovascular treatment than after endarterectomy. While the short-term benefit of surgery in stroke prevention is well recognized, the long-term benefits of the varied surgical CEA techniques in maintaining arterial patency remain elusive. The occurrence of carotid restenosis is a complex, not fully explained, process and it is potentially linked to the type of closure after arteriotomy. A meticulous closure after CEA is essential in the prevention of early and late restenosis and occlusion in both these techniques. When CEA is completed by the simple primary closure with fine sutures, it is often difficult to avoid carotid restenosis, especially at the distal end of the ICA in patients with small arteries. Alternatively, a way to preserve the internal carotid diameter and to minimize restenosis is to closing the arteriotomy with a patch. This allows making a closure of the bulk of the arteriotomy

*Corresponding author: Luigi luliano, MD, Sapienza University of Rome, Department of Medico-Surgical Sciences and Biotechnologies, Vascular Biology, Atherothrombosis \& Mass Spectrometry Lab, corso della Republica 79, 04100 Latina, Italy, Tel: +39 0773 1757231; Fax:+39 066229 1089; E-mail: antoniopacelli@tiscali.it

Received June 26, 2013; Accepted September 16, 2013; Published September 18,2013

Citation: Bertoletti G, Varroni A, Misuraca M, Massucci M, Pacelli A, et al. (2013) Carotid Artery Diameters, Carotid Endarterectomy Techniques and Restenosis. J Vasc Med Surg 1: 114 doi: 10.4172/2329-6925.1000114

Copyright: @ 2013 Bertoletti G, et al. This is an open-access article distributed under the terms of the Creative Commons Attribution License, which permits unrestricted use, distribution, and reproduction in any medium, provided the original author and source are credited. 
easier, but its distal portion may still be difficult to close without narrowing the artery. In contrast, when CEA is performed with eversion technique, there is no need to close the distal ICA by suture lines that are displaced to the more proximal. By avoiding the technical hazards of ICA closure, eversion technique has been shown in various studies to minimize the role of surgical closure in the occurrence of restenosis [7]. According to some authors, the natural history of recurrent carotid stenosis is generally benign [8,9] and only half of the patients who develop recurrent carotid artery stenosis can be expected to suffer from a recurrent ischemic event $[10,11]$. Other authors have acknowledged that the risk of stroke or progression to total occlusion is uncommon $[12,13]$. Most cases remain asymptomatic, but a number of patients with restenosis do become symptomatic and require an additional endarterectomy procedure [14]. The reported incidence of recurrent carotid stenosis varies widely and is influenced by evaluation techniques and criteria, the patient cohort studied, the duration of postoperative follow-up and reporting methods. The incidence of symptomatic carotid restenosis depends on the case series and ranges from $0 \%$ to $8.2 \%$, while asymptomatic carotid restenosis occurs in $1.3 \%$ to $37 \%$, but the true incidence of recurrent carotid stenosis is unknown [15]. It is also relevant to note that most studies have not reported restenoses of less than $50 \%$, usually because the available instrumentation has not been sufficiently accurate. In the first two years after surgery, the process of carotid restenosis is associated to myointimal hyperplasia that is likely to be dependent on platelet-mediated events. Platelets can rapidly adhere to the exposed collagen surface of the endarterectomized segment of carotid artery, and undergo collagen-induced aggregation and release of diverse bioactive mediators. Activated platelets have been shown to release a mitogenic factor, the platelet-derived growth factor (PDGF), that is capable of promoting migration and proliferation of smooth muscle cells into the subendothelial space [16,17]. In the early stages after surgery, the resulting myointimal hyperplasia differs histologically from primary atherosclerotic lesions. Two years after CEA, atherosclerosis superimposes and the lesions of late carotid restenosis are indistinguishable from primary atherosclerotic lesions. Risk factors for primary early restenosis include female sex, age, hyperlipidemia, hypertension, continued cigarette smoking, and history of cardiovascular disease [18-20]. The role of surgical technique as well as post-surgery anatomy of the carotid, which is dependent from caliber change after surgery, to minimize restenosis is under debate.

The purpose of this study was to prospectively examine the role of the varied surgical techniques on preoperative to postoperative variation in carotid dimensions, measured by color duplex imaging before and early after surgery. In addition, we aimed at investigating the role of carotid diameters and type of surgery towards later development of restenosis.

\section{Material and Methods}

175 patients eligible for CEA were enrolled in the study from October 2008 through December 2010. CEA was performed in symptomatic and asymptomatic patients with a carotid stenosis higher than $70 \%$ or $60 \%$, respectively. The surgical procedure used a standard access with a preparation of the internal carotid beyond the distal limit of the plaque. Carotid endarterectomy was performed using a traditional vascular surgery technique with particular attention to removal of the entire plaque and avoidance of intimal flaps. The reconstruction method (PR, PC or EV) was decided intra-operatively after assessment of the local anatomy, which included the length of the arteriotomy required to obtain a complete endarterectomy end point in ICA, the caliber and the angle of ICA, and the plaque extension. Patch reconstruction was performed by using a $6 \mathrm{~mm}$ Dacron patch. Conventional carotid duplex ultrasonography was performed with an HDI 1500 ultrasound system (Advanced Technology Laboratories, Bothell, WA) using a $7.5 \mathrm{MHz}$ linear array transducer. A single operator evaluated the carotid system before CEA, at hospital discharge, and at 3, 6 and 12 months after CEA. Carotid diameters were measured before and after surgery, before hospital discharge, at the following reference landmarks: common carotid (CC), carotid bulb (CB), proximal distal internal carotid artery (PICA), and distal internal carotid artery (DICA). Restenosis was defined as any occurrence of stenosis at 3, 6 and 12 months after CEA and was categorized as "minor" $(<50 \%)$ or "major" $(\geq 50 \%)$. The study procedure was developed according to the guidelines of the Ethic Committee, which approved the protocol, and the Helsinki Declaration of 1975. All subjects signed written informed consent.

Data were analyzed using SPSS for Windows (17th version). Statistical analysis was performed using Mann-Whitney Test and Kruskal-Wallis Test for continuous variables to compare mean values between respectively two and three independent groups. Wilcoxon Signed Rank Test for continuous variables was used to compare mean values between related groups. The $\chi^{2}$ test was used to compare categorical variables. Logistic regression analysis was used to calculate odds ratio of the variable of interest. Continuous variables are shown as mean \pm standard deviation. The level of statistical significance used throughout the study was defined as $\mathrm{p}<0.05$.

\section{Results}

Characteristics of the study population are reported in Table 1. The population enrolled in the study consisted of 175 patients, with a prevalence of male sex $(68 \%)$. The mean age was $80.7 \pm 6.71$ (mean \pm $\mathrm{SD}$ ) years in the whole population and was comparable between males and females. Apart from obesity, males carried a higher prevalence of cardiovascular risk factors compared to females. Concerning the surgical technique, PR was done in $42.9 \%$ of patients compared to $26.9 \%$ and $30.3 \%$ of patients undergoing PC and EV, respectively. The surgical technique applied to males and females differed consistently. PR, PC and EV were used in $42.9 \%, 26,9 \%$ and $30.3 \%$ of males, respectively. PR, PC and EV were used in $35.7 \%, 16.1 \%$ and $48.2 \%$ of females, respectively. Analysis of carotid diameter change as function of surgical technique, postoperative vs. preoperative, within each group is shown in Table 2. The technique of PR was associated with a significant increase in all of the carotid diameters after surgery (CC $+5 \%, \mathrm{CB}$ $+3.9 \%$, PICA $8.4 \%$, DICA $13.3 \%$ ). In contrast, PC was associated with a significant decrease in carotid diameters after surgery (CC $-2 \%, \mathrm{CB}$

\begin{tabular}{|l|c|c|c|c|}
\hline & All $(n=175)$ & Males $(n=119)$ & Females $(n=56)$ & $P$-value \\
\hline CV risk factors & & & & \\
\hline age & $80.70 \pm 6.71$ & $80.36 \pm 7.07$ & $81.43 \pm 5.87$ & 0.431 \\
\hline smoking & $52(29.7)$ & $39(32.7)$ & $13(23.2)$ & 0.455 \\
\hline diabetes & $72(41.1)$ & $41(34.4)$ & $31(55.3)$ & 0.059 \\
\hline obesity & $40(22.8)$ & $20(16.8)$ & $20(35.7)$ & 0.070 \\
\hline dyslipidemia & $114(65.1)$ & $75(63.0)$ & $39(69.6)$ & 0.577 \\
\hline hypertension & $159(90.8)$ & $109(91.5)$ & $51(91.0)$ & 1 \\
\hline Surgery & & & & \\
\hline Patch & $75(42.9)$ & $55(46.2)$ & $20(35.7)$ & 0.252 \\
\hline PC & $47(26.9)$ & $38(31.9)$ & $9(16.1)$ & 0.043 \\
\hline Eversion & $53(30.3)$ & $26(21.8)$ & $27(48.2)$ & 0.001 \\
\hline
\end{tabular}

PC: Primary Closure

Table 1: Characteristics of the study population 
-3.7\%, PICA -4\%, DICA -3.7\%). Concerning Eversion, CB (-2.7\%) and PICA (-2\%) diameters were significantly shorter after surgery, whereas CC and DICA did not show any difference in post-operative values compared to preoperative ones.

Preoperative and postoperative carotid diameters are shown in Table 3. The groups differed globally for preoperative carotid diameters. All preoperative diameters in the Patch group were lower than in PC and Eversion groups. The Eversion group and PC groups showed comparable diameters but PC had a higher CC diameter compared to Eversion group. After surgery, carotid artery diameters were comparable, i.e. they did not show any significant difference between the three groups of surgical techniques (Table 3 ).

No significant restenosis was observed either at 3 month or 6 months of follow up.

At 12 months follow up, color duplex imaging identified 16 overall restenoses of the internal carotid artery, including major restenosis occurring in 8 patients. Overall restenoses developed in $8 \%, 8.5 \%$ and $11.3 \%$ of patients with patch, PC, eversion endarterectomy, respectively. Major restenosis developed in $2.7 \%$ of those patients with patch closure, $4.3 \%$ of patients with primary closure, and $7.5 \%$ of patients with eversion

\begin{tabular}{|l|c|c|c|c|c|c|c|}
\hline & $\begin{array}{c}\text { Patch } \\
(n=75)\end{array}$ & $\begin{array}{c}P C \\
(n=47)\end{array}$ & $\begin{array}{c}\text { Eversion } \\
(n=53)\end{array}$ & $P$ & $\begin{array}{c}\text { Patch } \\
\text { vs } \\
P C\end{array}$ & $\begin{array}{c}\text { Patch } \\
\text { vs } \\
\text { Eversion }\end{array}$ & $\begin{array}{c}P C \\
\text { Vs } \\
\text { Eversion }\end{array}$ \\
\hline Preoperative values \\
\hline CC & $8.17 \pm 0.99$ & $9.01 \pm 1.16$ & $8.61 \pm 0.99$ & $<0.001$ & $<0.001$ & 0.019 & 0.035 \\
\hline CB & $8.44 \pm 0.87$ & $9.04 \pm 1.04$ & $8.74 \pm 1.29$ & 0.003 & 0.001 & 0.041 & $n s$ \\
\hline ProlCA & $6.39 \pm 0.91$ & $7.45 \pm 1.10$ & $7.33 \pm 1.15$ & $<0.001$ & $<0.001$ & $<0.001$ & $n s$ \\
\hline DisICA & $4.21 \pm 0.67$ & $5.08 \pm 1.26$ & $4.78 \pm 0.96$ & $<0.001$ & $<0.001$ & 0.001 & $n s$ \\
\hline Postoperative values \\
\hline CC & $8.58 \pm 1.06$ & $8.83 \pm 1.13$ & $8.72 \pm 1.11$ & $n s$ & -- & -- & -- \\
\hline CB & $8.77 \pm 1.06$ & $8.70 \pm 0.94$ & $8.50 \pm 1.61$ & $n s$ & -- & -- & -- \\
\hline ProlCA & $6.93 \pm 1.04$ & $7.15 \pm 1.06$ & $7.18 \pm 1.33$ & $n s$ & --- & -- & -- \\
\hline DisICA & $4.77 \pm 0.75$ & $4.89 \pm 0.92$ & $4.74 \pm 0.90$ & $n s$ & --- & -- & -- \\
\hline
\end{tabular}

PC: Primary Closure; CC: Common Carotid Artery; CB: Carotid Bulb; ProlCA: Proxymal Internal Carotid Artery; DisICA: Distal Internal Carotid Artery

Table 2: Comparison of preoperative and postoperative carotid diameters between patch, PC and eversion groups.

\begin{tabular}{|l|c|c|c|}
\hline & Preoperative values & Postoperative values & $P$ \\
\hline \multicolumn{4}{|c|}{ Patch $(n=75)$} \\
\hline CC & $8.17 \pm 0.99$ & $8.58 \pm 1.06$ & $<0.001$ \\
\hline CB & $8.44 \pm 0.87$ & $8.77 \pm 1.06$ & $<0.001$ \\
\hline ProICA & $6.39 \pm 0.91$ & $6.93 \pm 1.04$ & $<0.001$ \\
\hline DisICA & $4.21 \pm 0.67$ & $4.77 \pm 0.75$ & $<0.001$ \\
\hline \multicolumn{4}{|c|}{$P C(n=47)$} \\
\hline CC & $9.01 \pm 1.16$ & $8.83 \pm 1.13$ & 0.037 \\
\hline CB & $9.04 \pm 1.04$ & $8.70 \pm 0.94$ & $<0.001$ \\
\hline ProlCA & $7.45 \pm 1.10$ & $7.15 \pm 1.06$ & 0.001 \\
\hline DisICA & $5.08 \pm 1.26$ & $4.89 \pm 0.92$ & 0.002 \\
\hline \multicolumn{4}{|c|}{ Eversion $(n=53)$} \\
\hline CC & $8.61 \pm 0.99$ & $8.72 \pm 1.11$ & $n s$ \\
\hline CB & $8.74 \pm 1.29$ & $8.50 \pm 1.61$ & 0.002 \\
\hline ProlCA & $7.33 \pm 1.15$ & $7.18 \pm 1.33$ & 0.006 \\
\hline DisICA & $4.78 \pm 0.96$ & $4.74 \pm 0.90$ & $n s$ \\
\hline
\end{tabular}

PC: Primary Closure; CC: Common Carotid Artery; CB: Carotid Bulb; ProlCA: Proxymal Internal Carotid Artery; DisICA: Distal Internal Carotid Artery

Table 3: Preoperative and postoperative carotid diameters in patch, PC and eversion groups.

\begin{tabular}{|l|c|c|c|}
\hline \multicolumn{2}{|c|}{ No restenosis $(n=159)$} & Restenosis $(n=16)$ & $P$ \\
\hline Preoperative values \\
\hline CC & $8.51 \pm 1.06$ & $8.84 \pm 1.43$ & ns \\
\hline CB & $8.66 \pm 1.03$ & $9.07 \pm 1.55$ & ns \\
\hline ProlCA & $6.93 \pm 1.13$ & $7.48 \pm 1.24$ & ns \\
\hline DisICA & $4.62 \pm 1.01$ & $4.63 \pm 1.09$ & ns \\
\hline Postoperative values & & \\
\hline CC & $8.66 \pm 1.05$ & $9.03 \pm 1.54$ & ns \\
\hline CB & $8.65 \pm 1.19$ & $8.97 \pm 1.63$ & ns \\
\hline ProlCA & $7.05 \pm 1.09$ & $7.25 \pm 1.67$ & ns \\
\hline DisICA & $4.80 \pm 0.83$ & $4.77 \pm 1.02$ & ns \\
\hline
\end{tabular}

PC: Primary Closure; CC: Common Carotid Artery; CB: Carotid Bulb; ProlCA Proxymal Internal Carotid Artery; DisICA: Distal internal Carotid Artery

Table 4: Relationship between carotid diameters before and after CEA at discharge and overall restenosis.

endarterectomy. Notwithstanding an apparent increase in restenosis from $\mathrm{PC}$ to $\mathrm{PR}$ and to $\mathrm{EV}$, the rate of overall and major restenosis did not differ significantly between the three groups. In addition, logistic regression analysis showed that the type of surgical technique was not associated to an increased risk of either overall or major restenosis at 12 months.

Regardless of the type of surgical procedure used, there was no significant difference in all preoperative and postoperative carotid diameters between patients who developed overall restenosis and patients who did not develop it (Table 4). Similar findings were reported in patients who developed major restenosis.

Ultrasound analysis revealed a significantly higher rate of restenosis in female compared to male patients ( $10.7 \%$ vs $1.7 \%$ in males, $\mathrm{p}=0.014)$ Logistic regression analysis showed that female sex was associated with a higher risk of major restenosis (OR 6.9, 95\% CI 1.23 - 38.49). We did not observe any difference in postoperative carotid diameters between male and female patients by surgery techniques (not shown). As a higher incidence of restenosis in females has been attributed to the smaller vessel size and the presence of a small $(<4 \mathrm{~mm})$ internal carotid artery [21], we analyzed the incidence of restenosis using a cutoff point of $4 \mathrm{~mm}$ and did not find any statistically significant association with recurrent stenosis.

Both in males and females, there was no significant difference in all the preoperative and postoperative carotid diameters between patients who developed restenosis, either overall or major, and patients who didn't develop it.

\section{Discussion}

In our study, CEA with PR was associated with a significant increase in all the carotid diameters, while PC and EV produced a significant reduction in carotid artery diameters at discharge. In evaluating the potential association between restenosis and the type of surgery or carotid diameters, we found that the rate of restenosis during the first 12 months of follow up was comparable in the three surgical technique groups. Patients with restenosis compared to those without did not show any difference in the size of both preoperative and postoperative diameters.

Our results are in agreement with previous studies reporting that $\mathrm{PR}$ is associated to an increase [22-25] and PC to a decrease in carotid diameters [23,25]. Archie [22] reported an increase in ICA diameter by $20-30 \%$ for both vein and synthetic patched arteries. Golledge et al. [23] reported an increase in both DICA and CB diameters by patch repair. 
Archie [26] designed a study to determine whether common carotid and bulb diameters enlarged after application of saphenous patch. The authors found that both diameters increased postoperatively (by $87.3 \%$ and $19 \%$, respectively) and remained unchanged for as long 15 years. Morales et al. [24] investigated morphological and hemodynamic patterns of carotid stenoses treated by stenting or CEA with patch closure, and found that the PICA diameter increased by $36 \%$ and $110 \%$ in stented patients and patch closure patients, respectively.

In our eversion group, $\mathrm{CB}$ and PICA diameters significantly decreased but CC and DICA diameters remained essentially unchanged. Baan et al. [27] studied vessel wall and flow characteristics (diameter of $\mathrm{CB}$, strain, stiffness and turbulent flow) after eversion CEA and after CEA with Dacron patch and showed that after eversion the $\mathrm{CB}$ diameter was significantly smaller than after patch closure.

A number of studies have shown a decreased incidence of restenosis with patching, as compared to primary closure [28-30]. AbuRahma et al. [31] randomized a total of 339 CEA into five type of surgery - including primary closure, vein patch, saphenous patch and synthetic patch - and analyzed the long term clinical outcome and the incidence of restenosis. Patch closures resulted associated with a lower rate of perioperative stroke, and were superior in lowering the incidence of restenosis [31]. Naylor et al. [32] enrolled 276 patients who were randomly allocated to vein or thin-walled Dacron patch closure. Patch type had no influence on risk of ipsilateral stroke at 3 years, but Dacron patches were associated with a significantly higher incidence of recurrent restenosis after 3 years of follow up, with most occurring trough 6 to 12 months. A Cochrane systematic review [29] of seven trials including 1127 patients, undergoing 1307 operations, assessed the safety and efficacy of patch angioplasty compared to primary closure. The authors concluded that carotid patch angioplasty, either with a vein or a synthetic patch, may reduce the risk of carotid artery restenosis and subsequent ischemic stroke when compared to CEA with PC [29]. It should be taken into account that patch is associated with a higher risk of rupture, infection, or pseudoaneurysm formation [33].

It seems that patch angioplasty creates a wider arterial diameter than that achievable after PC, making any restenotic disease less significant, although the size of the internal carotid artery distal to the patch is always smaller [34]. On the basis of the increased size of the internal carotid artery after closure of the arteriotomy by patch, and assuming that deposition of neointima may be independent of the diameter of the artery, it has been suggested to use patch angioplasty for patients with small arteries as a selective approach, especially for female patients who are considered at particular risk of restenosis [21]. Other investigators have suggested that the increase in diameter by large sized patch may produce turbulent flow that may contribute to recurrent stenosis [35], although it has been reported that turbulence and disturbed flow patterns are more frequent in non-patched than in vein patched carotids [19]. A Cochrane systematic review has been conducted to evaluate the putative low risk of restenosis rate with eversion carotid endarterectomy in a total of 2465 patients and 2590 arteries [36]. The authors concluded that eversion CEA, compared to either primary closure or patch, may be associated with low risk of restenosis. However, reduced restenosis rates did not appear to be associated with clinical benefit in terms of reduced stroke risk, either perioperatively or later [36]. Other studies have shown a lower rate of restenosis with eversion compared to conventional CEA [37]. Crawford et al. [38] compared the rate of restenosis between primary closure and eversion 1-year after operation, in a study that included 155 PC and 135 EV. The authors found that restenosis rate was similar between eversion and patch, and underscored the role of biological phenomena rather than technicality of operation.

Surprisingly, although eversion CEA is not accompanied by an increase in arterial diameter, and the operated carotid artery resembles an unoperated-non-stenotic, artery, the resulting turbulent flow was not significantly different from that associated with patch closure [27].

In agreement with previous studies concerning the association between restenosis and gender [39,40], our study showed a higher percentage of restenosis in female compared to male patients. The higher incidence of restenosis in females has been attributed to a smaller vessel size, and an internal carotid artery diameter $<4 \mathrm{~mm}$ has been associated with a rate of recurrent stenosis almost three times that of the patient with a normal-sized artery [21]. Moreover, the fact that the carotid arteries in women are, on average, about $40 \%$ smaller than in men makes the operation technically challenging [41]. Conversely, in our study the presence of an ICA less than $4 \mathrm{~mm}$ diameter was not associated with recurrent stenosis $(\mathrm{p}>0.05)$, suggesting that the higher incidence of restenosis in females may be explained by other causes. It has been suggested that females are at high risk of restenosis because hormonal causes [42], or greater tendency toward carotid redundancy leading to kinking [43]. According to the present study, it is unlikely that the higher rate of restenosis in female could be dependent on cardiovascular risk factors since female patients carried lower cardiovascular risk factors compared to male patients.

In conclusion, we have reported that the type of surgical technique and postoperative diameters does not play a crucial role in the development of restenosis. The hope of decreasing restenosis by using anatomic characteristics or adopting certain surgery techniques is not warranted. Our study confirms that women are at high risk of restenosis that is an issue deserving further investigation to find and counteract causative factors.

\section{Acknowledgements}

This study was supported by grants from the Ministero dell' Università, Ricerca Scientifica e Tecnologica and from Sapienza University of Rome to LI.

\section{References}

1. Economopoulos KJ, Gentile AT (1999) Comparison of Carotid Endarterectomy Using Primary Closure, Patch Closure, and Eversion Techniques. Am J Surg 78: $505-510$

2. (1991) Beneficial effect of carotid endarterectomy in symptomatic patients with high-grade carotid stenosis. North American Symptomatic Carotid Endarterectomy Trial Collaborators. N Engl J Med 325: 445-453.

3. (1991) MRC European Carotid Surgery Trial: interim results for symptomatic patients with severe $(70-99 \%)$ or with mild $(0-29 \%)$ carotid stenosis. European Carotid Surgery Trialists' Collaborative Group. Lancet 337: 1235-1243.

4. (1995) Executive Committee for the Asymptomatic Carotid Atherosclerosis Study Endarterectomy for asymptomatic carotid artery stenosis. JAMA 273 1421-1428.

5. Hobson RW, Weiss DG, Fields WS Goldstone J, Moore WS, et al. (1993) Efficacy of carotid endarterectomy for asymptomatic carotid stenosis. The Veterans Affairs Cooperative Study Group. N Engl J Med 328: 221-227.

6. Bonati LH, Ederle J, McCabe DJ, Dobson J, Featherstone RL, et al. (2009) Longterm risk of carotid restenosis in patients randomly assigned to endovascula treatment or endarterectomy in the Carotid and Vertebral Artery Transluminal Angioplasty Study (CAVATAS): long-term follow-up of a randomised trial Lancet Neurol 8: 908-917.

7. Shah DM, Darling RC, Chang BB, Paty PS, Kreienberg PB, et al. (1998) Carotid endarterectomy by eversion technique: its safety and durability. Ann Surg 228: 471-478.

8. Carballo RE, Towne JB, Seabrook GR, Freischlag JA, Cambria RA (1996) An outcome analysis of carotid endarterectomy: the incidence and natural history of recurrent stenosis. J Vasc Surg 23: 749-753. 
Citation: Bertoletti G, Varroni A, Misuraca M, Massucci M, Pacelli A, et al. (2013) Carotid Artery Diameters, Carotid Endarterectomy Techniques and Restenosis. J Vasc Med Surg 1: 114 doi: 10.4172/2329-6925.1000114

9. Avramovic JR, Fletcher JP (1992) The incidence of recurrent carotid stenosis after carotid endarterectomy and its relationship to neurological events. $J$ Cardiovasc Surg (Torino) 33: 54-58.

10. de Borst GJ, Zanen P de Vries JP, van de Pavoordt ED, Ackerstaff RG, et al. (2008) Durability of surgery for restenosis after carotid endarterectomy. J Vasc Surg 47: 363-371.

11. Fluri F, Engelter ST, Wasner M, Stierli P, Merlo A, et al. (2008) The probability of restenosis, contralateral disease progression, and late neurologic events following carotid endarterectomy: a long-term follow-up study. Cerebrovasc Dis 26: 654-658

12. Lattimer CR, Burnand KG (1997) Recurrent carotid stenosis after carotid endarterectomy. Br J Surg 84: 1206-1219.

13. Healy DA, Zierler RE, Nicholls SC, Clowes AW, Primozich JF, et al. (1989) Long-term follow-up and clinical outcome of carotid restenosis. J Vasc Surg 10: 662-668

14. Gagne PJ, Riles TS, Jacobowitz GR, Lamparello PJ, Giangola G, et al. (1993) Long-term follow-up of patients undergoing reoperation for recurrent carotid artery disease. J Vasc Surg 18: 991-998.

15. Hertzer NR, Martinez BD, Benjamin SP, Beven EG (1979) Recurrent stenosis after carotid endarterectomy. Surg Gynecol Obstet 149: 360-364.

16. Harker LA, Schwartz SM, Ross R (1981) Endothelium and arteriosclerosis. Clin Haematol 10: 283-296.

17. Thomas M, Otis SM, Rush M, Zyroff J, Dilley RB, et al. (1984) Recurrent carotid artery stenosis following endarterectomy. Ann Surg 200: 74-79.

18. Dillavou ED, Kahn MB, Carabasi RA, Smullens SN, DiMuzio PJ (1999) Longterm follow-up of reoperative carotid surgery. Am J Surg 178: 197-200.

19. Archie JP Jr (1986) Prevention of early restenosis and thrombosis-occlusion after carotid endarterectomy by saphenous vein patch angioplasty. Stroke 17 : 901-905.

20. Volteas N, Labropoulos N, Leon M, Kalodiki E, Chan P, et al. (1994) Risk factors associated with recurrent carotid stenosis. Int Angiol 13: 143-147.

21. Ouriel K, Green RM (1987) Clinical and technical factors influencing recurrent carotid stenosis and occlusion after endarterectomy. J Vasc Surg 5: 702-706.

22. Archie JP Jr (1991) Early and late geometric changes after carotid endarterectomy patch reconstruction. J Vasc Surg 14: 258-266.

23. Golledge J, Cuming R, Davies AH, Greenhalgh RM (1996) Outcome of selective patching following carotid endarterectomy. Eur J Vasc Endovasc Surg 11: 458-463.

24. Morales MM, Anacleto A, Buchdid MA, Simeoni PR, Ledesma S, et al. (2010) Morphological and hemodynamic patterns of carotid stenosis treated by endarterectomy with patch closure versus stenting: a duplex ultrasound study. Clinics (Sao Paulo) 65: 1315-1323.

25. Archie JP Jr (1997) Geometric dimension changes with carotid endarterectomy reconstruction. J Vasc Surg 25: 488-498

26. Archie JP Jr (2002) Long-term geometric stability of saphenous vein patched carotid endarterectomy. J Vasc Surg 35: 131-136.

27. Baan J, Thompson JM, Reul GJ, Cooley DA, Brand R, et al. (1997) Vessel wall and flow characteristics after carotid endarterectomy: eversion endarterectomy compared with Dacron patch plasty. Eur J Vasc Endovasc Surg 13: 583-591.

28. Counsell C, Salinas R, Warlow C, Naylor R (2000) Patch angioplasty versus primary closure for carotid endarterectomy. Cochrane Database Syst Rev CD000160.

29. Bond R, Rerkasem K, AbuRahma AF, Naylor AR, Rothwell PM (2004) Patch angioplasty versus primary closure for carotid endarterectomy. Cochrane Database Syst Rev CD000160.
30. Mannheim D, Weller B, Vahadim E, Karmeli R (2005) Carotid endarterectomy with a polyurethane patch versus primary closure: a prospective randomized study. J Vasc Surg 41: 403-407.

31. AbuRahma AF, Robinson PA, Saiedy S, Kahn JH, Boland JP (1998) Prospective randomized trial of carotid endarterectomy with primary closure and patch angioplasty with saphenous vein, jugular vein, and polytetrafluoroethylene: long-term follow-up. J Vasc Surg 27: 222-232.

32. Naylor R, Hayes PD, Payne DA, Allroggen H, Steel S, et al. (2004) Randomized trial of vein versus dacron patching during carotid endarterectomy: long-term results. J Vasc Surg 39: 985-993.

33. Awad IA, Little JR (1989) Patch angioplasty in carotid endarterectomy Advantages, concerns, and controversies. Stroke 20: 417-422.

34. Harris RA, Stow N, Fisher CM, Neale ML, Appleberg M (2003) Carotid redo surgery: both safe and durable. ANZ J Surg 73: 1000-1003.

35. Fietsam R, Ranval T, Cohn S, Brown OW, Bendick P, et al. (1992) Hemodynamic effects of primary closure versus patch angioplasty of the carotid artery. Ann Vasc Surg 6: 443-449.

36. Cao PG, de Rango P, Zannetti S, Giordano G, Ricci S, et al. (2001) Eversion versus conventional carotid endarterectomy for preventing stroke. Cochrane Database Syst Rev CD001921.

37. Cao P, de Rango P, Zannetti S (2002) Eversion vs conventional carotid endarterectomy: a systematic review. Eur J Vasc Endovasc Surg 23: 195-201.

38. Crawford RS, Chung TK, Hodgman T, Pedraza JD, Corey M, et al. (2007) Restenosis after eversion vs patch closure carotid endarterectomy. J Vasc Surg 46: 41-48.

39. Hugl B, Oldenburg WA, Neuhauser B, Hakaim AG (2006) Effect of age and gender on restenosis after carotid endarterectomy. Ann Vasc Surg 20: 602-608.

40. Reina-Gutiérrez T, Serrano-Hernando FJ, Sánchez-Hervás L, Ponce A Vega de Ceniga M, et al. (2005) Recurrent carotid artery stenosis following endarterectomy: natural history and risk factors. Eur J Vasc Endovasc Surg 29: 334-341.

41. Rothwell PM, Slattery J, Warlow CP (1997) Clinical and angiographic predictors of stroke and death from carotid endarterectomy: systematic review. BMJ 315 1571-1577.

42. Lane JS, Shekherdimian S, Moore WS (2003) Does female gender or hormone replacement therapy affect early or late outcome after carotid endarterectomy? J Vasc Surg 37: 568-574

43. Cossman DV, Treiman RL, Foran RF, Levin PM, Cohen JL (1980) Surgical approach to recurrent carotid stenosis. Am J Surg 140: 209-211. 